
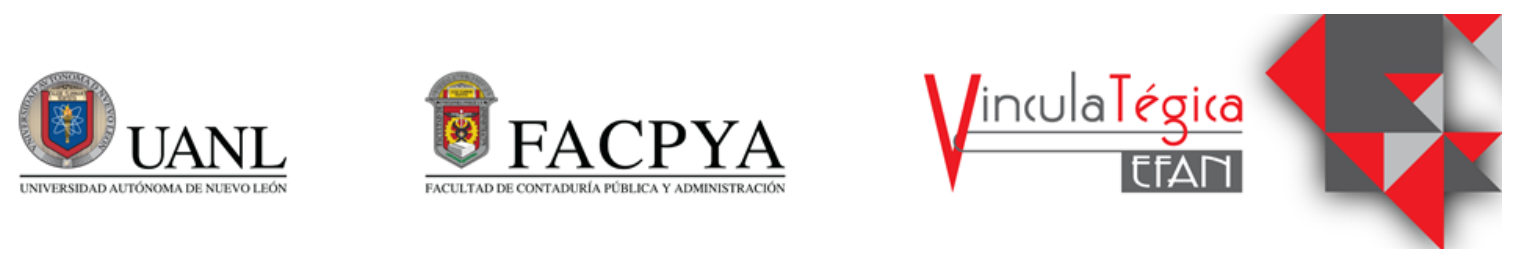

\title{
Análisis del clima organizacional en el sector público durante Covid-19
}

\author{
José Esteban Mendoza Flores ${ }^{1}$, Ernesto Casas Cárdenas ${ }^{2}$ y Yesenia Sánchez Tovar ${ }^{3}$ \\ ${ }^{I}$ Facultad de Derecho y Ciencias Sociales Victoria de la Universidad Autónoma de Tamaulipas, \\ emflores@docentes.uat.edu.mx, Centro Universitario Campus Victoria, Cd. Victoria, Tamaulipas, México \\ C.P.87120, 834-318-1800 ext. 2712 \\ ${ }^{2}$ Facultad de Derecho y Ciencias Sociales Victoria de la Universidad Autónoma de Tamaulipas, \\ ecasas@docentes.uat.edu.mx, Centro Universitario Campus Victoria, Cd. Victoria, Tamaulipas, México C.P.87120, \\ 834-318-1800 \\ ${ }^{3}$ Facultad de Comercio y Administración Victoria de la Universidad Autónoma de Tamaulipas, \\ yesanchez@docentes.uat.edu.mx, Centro Universitario Campus Victoria, Cd. Victoria, Tamaulipas, México \\ C.P.87120, 834-318-1800
}

Información del artículo revisado por pares

Fecha de aceptación: junio-2021

Fecha de publicación en línea: diciembre-2021

DOI: https://doi.org/10.29105/vtga7.1-103

\section{Resumen}

Se ha identificado que el clima organizacional juega un papel clave en el crecimiento de las organizaciones y su estudio a profundidad incide de manera directa en el espíritu de servicio de la organización tanto pública como privada. La presente investigación realiza un planteamiento conceptual a partir de diferentes ópticas y se identifica la evolución del constructo y se refieren a las dimensiones primordiales que construyen el clima organizacional en tiempos del Covid-19 con el fin identificar las dimensiones que tienen mayor peso en el mismo en una institución pública que se realizó un estudio empírico con 982 servidores públicos y permitió concluir que las dimensiones de Liderazgo y Supervisión, Apertura Organizacional, Presión Laboral, Cohesión y Claridad Organizacional.

Palabras clave: Clima organizacional, Sector público, Covid-19.

\begin{abstract}
Organizational climate has been identified as playing a key role in the growth of organizations and its in-depth study directly affects the spirit of service of both public and private organizations. In this sense, in this research a conceptual approach is reflected from different perspectives and the evolution of the construct is identified, also, they refer to the primary dimensions that the organizational climate builds in times of COVID-19, in order to identify the dimensions that have greater weight in it in a public institution, therefore, an exploratory study was carried out with 982 public servants, the above allowed to conclude that the dimensions of Leadership and Supervision, Organizational Openness, Work Pressure, Cohesion and Organizational Clarity, have greater relevance in the analyzed organizational climate.
\end{abstract}

Keywords: Organizational climate, Public sector, Covid-19.

JEL: D29, E24, H83, J23, J24 


\section{Introducción}

Hoy en día todas las organizaciones están

preocupadas en lograr adaptarse a los entornos turbulentos en donde subsisten, esta preocupación no exime a las organizaciones públicas. No obstante, son las organizaciones públicas quienes se encuentran azotados por una crisis que abarca desde problemas económicos, estructurales, pero sobre todo de credibilidad y confianza por parte de sus usuarios (Christensen y Laegreid, 2002). Aunado a las problemáticas expuestas, las organizaciones viven la crisis sanitaria derivada de la pandemia del COVID-19, en respuesta a esta pandemia, debieron adaptarse rápidamente a una nueva realidad, cambiando sus políticas y hasta las rutinas de trabajo (Nicola et al., 2020). En ese sentido se debe plantear una pregunta ¿Cuáles son las dimensiones del clima organizacional mejor valoradas por los empleados en las organizaciones públicas durante la pandemia del COVID-19?

El clima dentro de las organizaciones es un elemento determinante para la mejora en los procesos de gestión y operación, lo cual incide en la calidad, a través de una mejor adaptación al cambio y la innovación (Martín Bris, 2000). Asimismo, el clima organizacional ofrece una oportunidad para desarrollar el recurso humano a través de aspectos de cambio de actitudes y comportamientos, que se espera sean capaces de ajustar los desafíos actuales y futuros (Suprapti et al., 2020).

Brown y Leigh (1996), han destacado que los trabajadores de toda organización pueden ser capaces de desarrollar ventajas competitivas, esto siempre que se encuentre en un adecuado clima organizacional. Esta idea es reforzada por Velázquez, et al. (2014), al explicar que un clima organizacional adecuado tiene efectos favorables en el personal y consecuentemente coadyuva el cumplimiento de los objetivos organizacionales. Dentro de la literatura se ha demostrado que el clima organizacional es un marco útil para entender cómo las medidas organizativas pueden modificar el comportamiento individual (Griffin \& Curcuruto,2016; Kuenzi \& Schminke,2009; Schneider et al., 2013).

Las organizaciones del sector público al igual que las del privado, tienen la necesidad de cumplir con sus objetivos, lo que permite ofrecer servicios de calidad. Por tal motivo, conocer los elementos que conllevan a un buen clima organizacional resulta de interés general para la administración pública.

En este sentido el objetivo de este documento es identificar aquellas dimensiones que tienen un mayor peso en el clima organizacional en una institución pública dedicada a brindar asistencia social en tiempos de pandemia y con ello responder a la pregunta planteada. Para el cumplimento de este objetivo, el documento se estructura de la forma siguiente. En la siguiente sección se presenta una revisión de la evolución del clima organizacional. El tercer apartado explica los estudios que han identificado las dimensiones del clima organizacional. Enseguida se presenta la metodología y resultados 
encontrados. Finalmente, se presentan algunas conclusiones respecto al estudio realizado.

\section{Evolución y definición del clima organizacional}

Según Gan (2007), los resultados del estudio realizado por Elton Mayo entre 1925 y 1932 en los talleres de la Western Electric Company en Chicago, se pueden considerar como la primera aportación en la historia del clima organizacional, toda vez que se pone de manifiesto el valor de las relaciones humanas con variables de percepción axiomáticas de la productividad y la satisfacción del recurso humano que compone el clima organizacional, como los sentimientos de los trabajadores, los intereses colectivos, las condiciones de trabajo y el sentido de pertenencia.

De la misma manera, en el trabajo de Lewin et al (1939) sobre el estudio del comportamiento de niños en función del tipo de liderazgo con el que eran dirigidos en un campamento, se gestó el concepto de clima social como una manera de capturar el tipo de clima que se creó en el grupo de estudio, es decir, para referirse en lo particular a los diversos patrones de comportamiento que surgían al interior del colectivo analizado, el cual sirvió como primera referencia para el concepto de clima organizacional.

Durante la década de los 40 y hasta finales de los 60's las investigaciones sobre clima organizacional se centraron en estudios de caso abocándose más al desarrollo del clima desde la perspectiva de la psicología organizacional, existía una amplia historia conceptual y los principales investigadores centraban sus estudios en las impresiones del hombre que rodeaba la eficacia de la organización, especialmente en el papel del liderazgo y del sistema social en su conjunto en el cual se desempeñaban los trabajadores (Tiffin y McCormick 1965; Viteles 1953; Argyris 1958, y; Litwin y Stringer 1968).

No obstante, Schneider (2013) identifica que las investigaciones sobre clima organizacional que aplicaron métodos cuantitativos formales comenzaron alrededor de la década de los 70 , pero estos estudios se distinguieron por las diversas definiciones en la medición del tema, casi ninguno con orientación conceptual a las primeras medidas diseñadas para evaluarlo.

Con respecto a esos estudios, una serie de publicaciones (Hellriegel y Slocum, 1974, y Payne y Pugh, 1976) aportaron un marco de referencia que definió el estudio del clima organizacional desde tres enfoques diferentes, el primero de ellos está basado en medir la percepción de atributos individuales del trabajador; el segundo sustenta que se debe medir la percepción de los atributos de la organización, y; por último está medir de manera agregada los atributos organizacionales, es decir, combinar mediciones de percepción con otras más objetivas. Derivado de estas perspectivas, se determinó que el primer enfoque aborda el clima psicológico y el segundo y tercero plantea el estudio del clima organizacional.

A causa de la diversidad de enfoques y metodología de naturaleza cualitativa, se acentuaron las críticas de las limitaciones de estas técnicas para el estudio de los constructos del 
clima organizacional. De ahí que Pettigrew (1979) propone el concepto de cultura organizacional como alternativa, considerándola como doctrinas o valores compartidos.

No obstante, debido al aumento que estaba adquiriendo el estudio de la cultura organizacional y a la confusión presente entre ambos constructos, las investigaciones sobre el clima organizacional se vieron disminuidas en la década de los 80. Por consiguiente, los investigadores sobre el clima centraron sus estudios en mayor medida para identificar la formación del clima organizacional, fundamentado en lo que llamaron el proceso de atracción-selección-retención, poniendo al descubierto la relación dinámica para la formación del clima, la cual es correspondida por los cambios que experimentan los miembros de la organización aunado al proceso de sociabilización (Schneider y Reichers 1983; Schneider 1987, y; Reichers 1987).

Durante la década de los 90, los investigadores centraron sus estudios en definir las similitudes y diferencias entre los constructos de clima y cultura organizacional, por un lado, Pettigrew (1990) revisó la literatura para identificar y argumentar las semejanzas y discrepancias entre ambos conceptos, planteando el uso de la cultura para futuros análisis de la organización. Sin embargo, Reichers y Schneider (1990) evalúan la construcción teórica del estudio del clima y la cultura organizacional, planteando que la evolución de un constructo necesariamente debe transitar por tres fases, la primera de ellas es la introducción y elaboración; la segunda es la evaluación y argumentación, y; la tercera es la consolidación.

Para concluir con la evolución del clima organizacional en el año 2000 se continuaron realizando estudios con el objetivo de analizar la similitud del clima y la cultura organizacional. Se analizaron los métodos de investigación utilizados en cada uno de ellos, proveyendo una vasta descripción sobre investigaciones, teorías y prácticas en ambos campos, con análisis empíricos y simultáneos de ambos constructos.

Ahora bien, como se ha visto en los párrafos anteriores, el clima organizacional es una construcción teórica desarrollada para comprender la forma en que los individuos perciben y describen sus entornos laborales, tanto en las empresas privadas como en instituciones públicas.

De acuerdo con Schneider (1975), el estudio del clima organizacional sienta sus bases en dos supuestos claves. El primero de ellos plantea que las personas intentan aprender el orden en su entorno para crear el orden a través del pensamiento, en tanto que el segundo supuesto parte de la premisa que los seres humanos aprueban y/o intentan crear orden en su entorno para adaptar eficazmente su comportamiento al ambiente de trabajo. En lo que respecta a la primera suposición se asocia con la escuela psicológica de Gestalt, mientras que la segunda hipótesis es representativa de los tipos de preguntas asociadas con la escuela del funcionalismo. 
Cabe destacar que, desde la evolución del constructo del clima organizacional, no hay un consenso general para establecer una definición única, aunque el trabajo de Moran y Volkwein (1992) aporta un modelo conceptual que permite demarcar las distintas definiciones aportadas a esa construcción teórica. En ese sentido, estos autores proponen cuatro perspectivas, esto es, la perceptual, la estructural, la interactiva y la cultural.

Con respecto a la primera, se puede considerar que el trabajo desarrollado por Litwin y Stringer (1968) fue el primero en conceptualizar el clima organizacional dentro de esa perspectiva, considerando a la percepción como el componente más importante del clima. Del mismo modo, Hall y Schneider (1972) consideran que las percepciones globales de los individuos que conforman una organización son la base para la construcción del clima organizacional.

Por su parte, Dastmalchian et al (1986) proponen que el clima organizacional es el concepto central que expresa el ambiente laboral, que interviene directamente en la motivación, la satisfacción y el comportamiento de los trabajadores dentro de la organización. Finalmente, Goncalves (1997) reflexiona que esta perspectiva del clima ha demostrado tener un mayor beneficio al considerar las percepciones que los individuos de las organizaciones tienen sobre las estructuras y procesos que ocurren en el ambiente laboral.

Con relación a la representación estructural, el clima organizacional representa una serie de tipologías objetivas perdurables y de fácil medición que diferencia una organización de otra (Forehand y Gilmer 1964). Del mismo modo, Hilton (1966) define que el clima está compuesto por particularidades que pueden distinguir una organización de otra y estos rasgos a su vez influyen en el comportamiento de los individuos que conforman las instituciones. Por su parte, Litwin y Stringer (1968) consideran que midiendo el clima organizacional se puede determinar cómo es percibida la organización, ya que el clima funcionaria como una especie de filtro por el cual pasan expresiones objetivas (estructura, liderazgo, entre otros).

Para terminar, al analizar las definiciones desde la perspectiva interactiva, la cual ha sido la menos desarrollada de los enfoques expuestos, Taguiri (1968) considera que el clima organizacional se compone de una serie de elementos, tales como el entorno, las interacciones de las personas y los grupos, su cultura, entre otros. Por su parte, para Hall y Schneider (1972) el clima organizacional pone de manifiesto las relaciones entre las tipologías personales y organizacionales.

Para nuestro estudio vamos a tomar la definición propuesta por Chiavenato (2000), referente al estado del clima organizacional percibido o experimentado por los trabajadores y que influye en su comportamiento.

Cabe destacar que, el análisis del clima organizacional adquiere relevancia porque permite mejorar el espíritu de trabajo a través del conocimiento de las satisfacciones e 
insatisfacciones del recurso humano, estas pueden modificarse implementando planes que permitan que la organización mejore (Segredo 2013, y; García 2009). Un clima organizacional positivo colabora en el éxito de la organización y al compromiso de los colaboradores hacia ésta (Toro Álvarez y Sanín Posada, 2013).

Ahora bien, como ya se había establecido, actualmente las organizaciones a nivel nacional e internacional están conllevando el impacto de la crisis sanitaria originada por el COVID-19, lo que, indudablemente está afectando al clima organizacional, tanto en el sector privado como en el público, por lo cual se vuelve urgente y necesario conocer y medir el clima organizacional en que los colaboradores se están desenvolviendo.

\section{Dimensiones del clima organizacional}

Las llamadas dimensiones del clima, se refiere a las características que son objeto de medida dentro de una organización, ya que influyen en el comportamiento de los individuos. Diferentes propuestas de dimensiones para el estudio del clima organizacional han sido estudiadas.

El primer autor en abordar los elementos del clima organizacional fue Litwin y Stringer (1968), de acuerdo con ellos, el clima observa nueve elementos:

- Estructura: Hace referencia a las políticas, normas, procedimientos y reglas bajo las cuales deben regirse los empleados al interior de la organización, así como los factores motivacionales para su desempeño, ya que sea positiva o negativamente.

- Responsabilidad individual: Se refiere a la autonomía que pueda sentir el individuo para desenvolverse y tomar decisiones.

- Cooperación: Se refiere al grado de apoyo entre los empleados, ya sea de su mismo nivel jerárquico o cualquier nivel vertical de la estructura.

- Recompensas y castigos: percepción del individuo respecto a las remuneraciones justas y equitativas que recibe por las actividades que lleva a cabo dentro de la organización, o por el contrario los castigos o sanciones a las que puede ser acreedor.

- Conflicto y tolerancia: Dentro de la cultura organizacional existe una diversidad de opiniones, comportamientos y actitudes a los cuales debe ser tolerante y encontrar la mejor forma de manejarlos, de tal forma que se eviten los conflictos.

- Estándares y expectativas: Se refiere al grado en que los individuos toman en cuenta las normas o reglamentos para desempeñarse y la productividad que llegan a tener dentro de la organización.

- Identidad organizacional y lealtad del grupo: Es el grado de pertenencia o identificación con su empresa y por lo tanto se sienta comprometido con el cumplimiento de los objetivos.

- Riesgos y desafíos: Las organizaciones toman riesgos en ocasiones, lo que muchas veces representa desafíos que podrían ser 
positivos o negativos para éstas, en cuanto al cumplimiento de sus metas.

- Relaciones: Tanto entre las personas del mismo nivel jerárquico, como jefe y subordinado, es una característica esencial para que haya un buen clima en la organización.

Otros estudios como los de Brunet (2005) implican que el clima se agrupa en cuatro dimensiones:

- Autonomía individual: Engloba los aspectos de la responsabilidad e independencia que puede llegar a tener un individuo, al momento de tomar decisiones.

- Grado de estructura que impone el puesto: Mide el grado de transferencia de información respecto a que las actividades y procedimientos a seguir para realizarlas por parte de los superiores.

- Recompensas: Se refiere a las remuneraciones monetarias que reciben los empleados como recompensa por su trabajo, así como las promociones que se dan en la empresa por el buen desempeño de algún trabajador.

- Consideración, agradecimiento y apoyo: Se refiere a las relaciones y grado de compañerismo, entre los elementos de la organización.

Uno de los autores más referenciados en el estudio del clima, fue Likert (1976), quien llegó a considerar dicho concepto a partir de ocho dimensiones:
- Métodos de mando: La forma en que los líderes influyen en sus empleados, para que exista una buena relación.

- Características de las fuerzas motivacionales: Los procedimientos que se llevan a cabo para responder a las necesidades o inquietudes de los empleados y así estos se sientan motivados en su trabajo.

- Características de los procesos de comunicación: Se refiere a las técnicas o tipos de comunicación que se ejercen en la organización.

- Características de los procesos de influencia: La importancia que se le infiere a la buena relación, que debe existir entre los superiores y subordinados, para que se logren los objetivos de la organización.

- Características de los procesos de toma de decisiones: El manejo de la información a través de los diferentes niveles jerárquicos, así como el reparto de las funciones.

- Características de los procesos de planeación: La manera en que se establecen los objetivos o directrices que tomará la organización.

- Características de los procesos de control: La distribución de los tramos de control entre la estructura de la organización.

- Los objetivos de rendimiento y perfeccionamiento: Se refiere al continuo control del cumplimiento de lo que se tiene planeado en cierto periodo de tiempo.

Una vez mencionadas las distintas clasificaciones de dimensiones utilizadas por 
diversos autores, se puede observar que el concepto de clima organizacional es un concepto multidimensional, que varía entre un autor y otro.
Para la presente investigación, consideramos las dimensiones siguientes:

Tabla 1 Dimensiones del clima organizacional

\begin{tabular}{|c|c|}
\hline Dimensión & Definición \\
\hline Liderazgo & $\begin{array}{l}\text { Se refiere a la percepción del empleado sobre la capacidad que tiene un individuo } \\
\text { para influir en la manera de pensar, de ser o actuar de las personas o en equipos de } \\
\text { trabajo establecidos, con la finalidad de que trabajen en el logro de sus metas y } \\
\text { objetivos previamente establecidos a través de la destreza de orientar y convencer. }\end{array}$ \\
\hline Supervisión & $\begin{array}{l}\text { Se refiere a la percepción del empleado sobre el contacto que tiene con su jefe directo } \\
\text { referente a tratar temas de trabajo, la preocupación por la calidad del trabajo y la } \\
\text { evaluación correcta en el desempeño de sus funciones. }\end{array}$ \\
\hline Cohesión & $\begin{array}{l}\text { Se refiere a como el empleado percibe las relaciones entre los compañeros al interior } \\
\text { de la organización, la presencia de un ambiente que propicie interrelaciones } \\
\text { amigables y de confianza que abonen en el compromiso personal con los demás para } \\
\text { la realización de las tareas. }\end{array}$ \\
\hline Ergonomía & $\begin{array}{l}\text { Se refiere a la percepción del empleado referente a como la organización buscan } \\
\text { generar condiciones óptimas y confortable al disminuir los contaminantes } \\
\text { ambientales y las condiciones físicas que prevalecen en el puesto de trabajo. }\end{array}$ \\
\hline Presión Laboral & $\begin{array}{l}\text { Se refiere a como el empleado percibe la relación entre los estándares para medir el } \\
\text { desempeño y las cargas de trabajo, estrés y sentimientos de no descanso. }\end{array}$ \\
\hline Claridad Organizacional & $\begin{array}{l}\text { Se refiere a la percepción del empleado referente a como identifica que la } \\
\text { organización es clara en establecer las metas y objetivos. }\end{array}$ \\
\hline
\end{tabular}

Fuente: Elaboración propia a partir de Koys \& Decottis, (1991)

Por lo anterior y a partir de la revisión de la literatura se plantea la hipótesis siguiente:

H1: Las dimensiones que componen el clima organizaciones en una institución pública son: Liderazgo, Supervisión, Cohesión, Ergonomía, Presión Laboral y Claridad Organizacional.

\section{Método}

Con la finalidad de identificar las dimensiones que tienen mayor peso en el clima organizacional en una institución pública del Gobierno del Estado de Tamaulipas, se realiza la presente investigación de enfoque cuantitativo, de alcance exploratorio, al indagar el entorno interno de la institución. $\mathrm{Su}$ diseño es cuasiexperimental puesto que no se tiene control de las variables, solo se observó la realidad a partir de la recolección de datos, esto sin pretender algún cambio de esta. Así mismo es un estudio transversal, ya que solamente se estudió la percepción de los empleados de la institución en un periodo determinado (Münch \& Ángeles, 2007; Hernández, Fernández \& Baptista, 2006).

\section{Instrumento y recolección de datos}

La recolección de datos para el estudio estuvo centrada en la aplicación de un cuestionario a una muestra de 1034 servidores públicos, logrando de esta forma 982 cuestionarios validados. $\mathrm{La}$ recolección de la información se llevó a cabo en el mes de abril de 2021. 
El cuestionario aplicado fue desarrollado a partir de la revisión de la literatura de clima organizacional. Dicho instrumento fue diseñado considerando el desarrollado por Freire et al. (2011), Patterson et al. (2005), Koys y Decottis (1991), Chiang et al (2008) y Brown y Leigh (1996). Logrando una medición del clima organizacional considerando seis dimensiones Liderazgo, Cohesión, Claridad organizacional, Ergonomía, Supervisión y Presión Laboral.

Cada dimensión del clima organizacional estuvo medida por 5 ítems, logrando un total de 30 ítems. El instrumento fue medido a través de una escala de 5 puntos donde el servidor público debe posicionarse en aquella actitud que la describe acercándose a los polos 1 o 5 de la escala, donde 1 representa un totalmente en desacuerdo hasta el 5 que representa un totalmente de acuerdo. Debe indicarse que la dimensión de presión laboral se mide a través de una escala inversa. El cuestionario además recoge datos socioeconómicos de la edad, género, antigüedad, tipo de contrato y nivel jerárquico.

\section{Técnica}

Las técnicas estadísticas utilizadas para el tratamiento de los datos fueron en primer lugar el análisis de fiabilidad a través del Alpha de Cronbach. Para comprobar la relación entre las variables se utilizó el análisis de Correlación de Pearson. Para el tratamiento de la información se utilizó el paquete estadístico SPSS en la versión 21.

\section{Resultados}

\section{Perfil de los encuestados}

A través del análisis descriptivo de frecuencias se identificaron las principales generalidades de los encuestados que integran la institución sujeta de estudio, destacando: la edad, género, antigüedad, tipo de contrato y nivel jerárquico de los encuestados como a continuación se muestra:

Del total de los encuestados el 56\% son mujeres y el $44 \%$ son hombres. En lo que respecta a la edad tenemos que del total de encuestados el $33 \%$ tiene entre 35 y 39 año, el 19\% entre 40 y 44 , el $15 \%$ entre 25 y 29 años, el $12 \%$ tiene entre 45 y 49 años, los rangos de entre 30 y 34 años y 55 a 59 años tienen un $8 \%$ respectivamente, el rango de 50 a 54 años tiene un $4 \%$ y el $2 \%$ corresponde a los servidores públicos que tienen 60 años y más.

Gráfica 1. Edad de los encuestados 


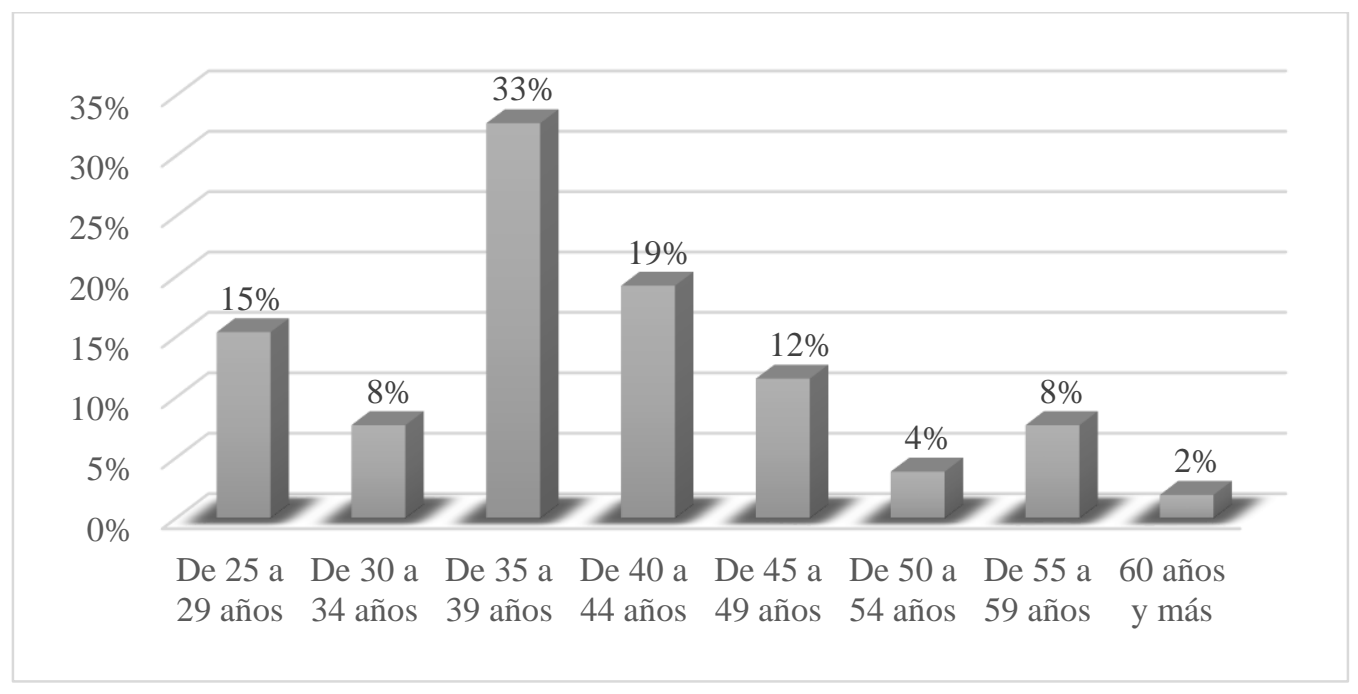

Como se observa en la gráfica 2, el $38 \%$ de los encuestados tienen entre 9 a 16 años de antigüedad, el 29\% tiene menos de 1 año de antigüedad, los encuestados en el rango de 17 a
24 tienen un $15 \%$ de antigüedad, el $13 \%$ corresponde a los que tienen más de 25 años de antigüedad y el rango de 1 a 8 años tienen el $4 \%$.

Gráfica 2. Antigüedad de los encuestados

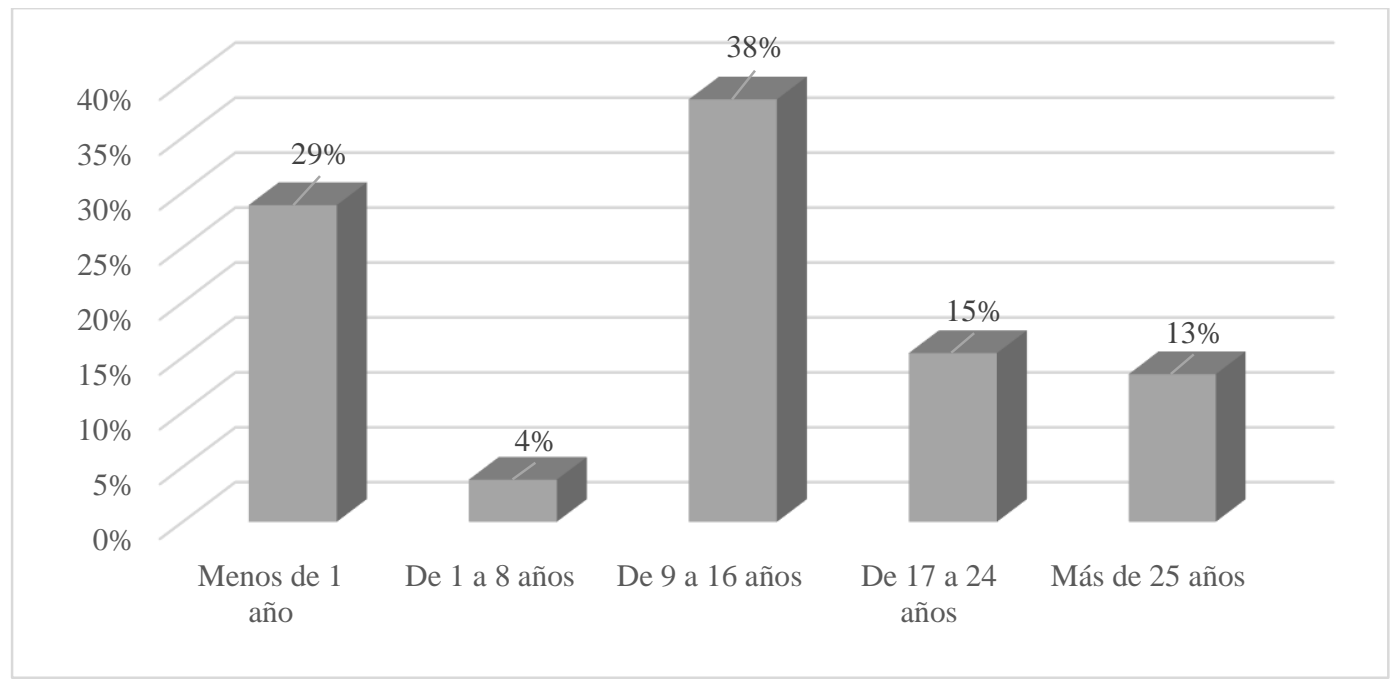

de 160 a 200, el $4 \%$ corresponde al nivel de 100 a

Se puede apreciar en la gráfica 3 que del total de 129 y del nivel de 70 al 99 tienen un $2 \%$. encuestados el 77\% tienen un nivel de 130 al 159, el $17 \%$ de los encuestados cuentan con un nivel

Gráfica 3. Clave o nivel del puesto que desempeña en la institución 


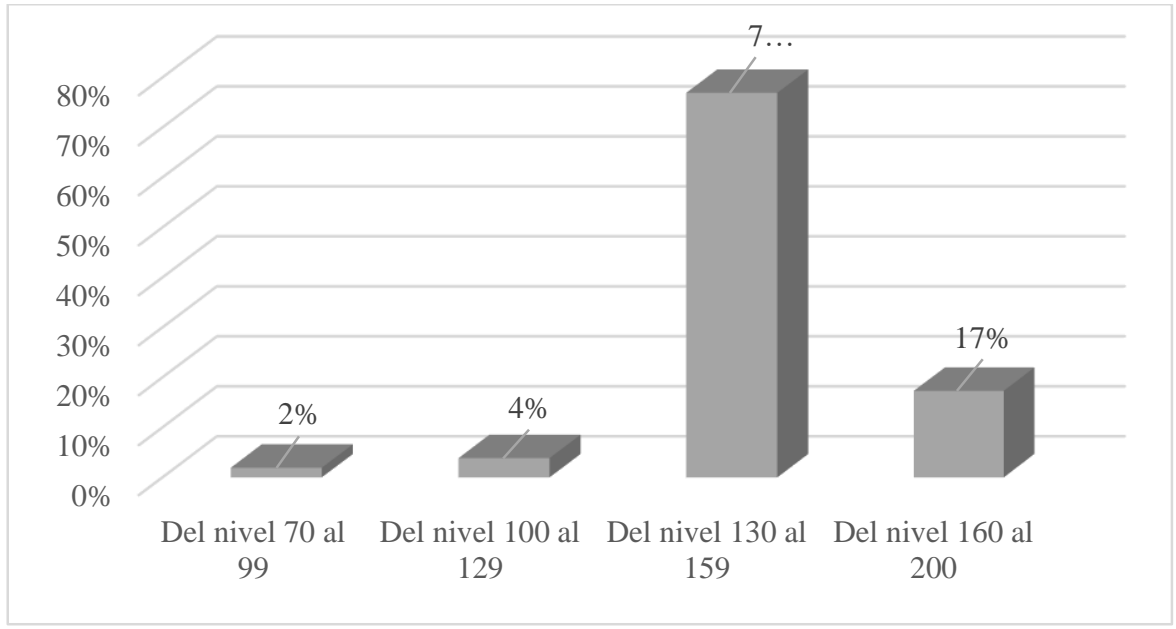

Por lo que respecta al tipo de contratación de los encuestados, los que cuentan con contrato por

base y confianza tienen un $48 \%$ respectivamente y solo el $4 \%$ tienen contrato extraordinario.

Gráfica 4. Tipo de contratación

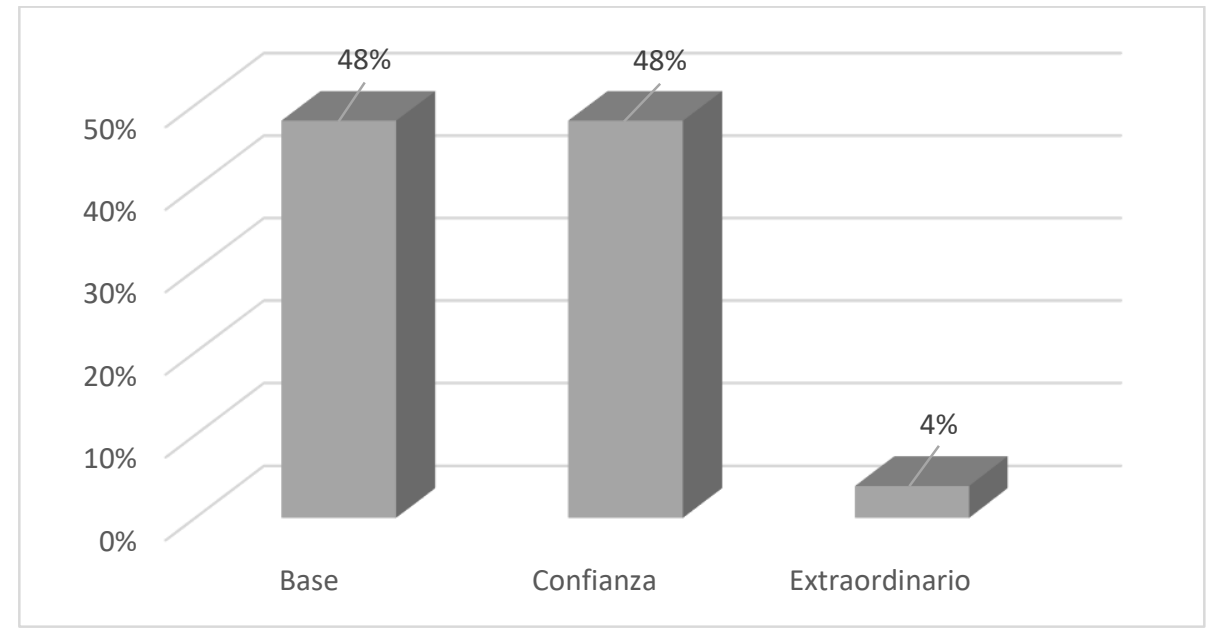

Fiabilidad de la escala de clima organizacional.

Para comprobar las dimensiones que se identifican en la medición del clima organizacional se realizó un análisis factorial considerando los 50 ítems de la variable clima, al realizarlo se obtuvo de manera final una Medida de adecuación muestral de Kaiser-Meyer-Olkin de 0.955 y un test de esfericidad de Barlett con un chi cuadrado de 21224.87 con una significancia estadística de 0.001, lo cual implica que el uso de la técnica factorial para la identificación de grupos de variables que comparten varianza en común es adecuada. Se utilizó el método de Máxima verosimilitud y la rotación varimax.

La Tabla 2 muestra la validez de las dimensiones y las cargas factoriales de cada uno 
de los ítems considerados en las dimensiones del clima organizacional.

En primer lugar, debe indicarse que las dimensiones definidas del clima explicaron un $64.63 \%$ de la varianza. Sin embargo, de las 10 dimensiones consideradas para el análisis del clima, de acuerdo con el Análisis factorial, solamente se identificaron 5 dimensiones, puesto que se eliminaron ítems que tenían cargas factoriales insatisfactorias al ser menores del
0.500 (Hair et al., 2010). Por lo que se lograron definir solamente las dimensiones de Liderazgo, Cohesión, Ergonomía, Presión Laboral y Claridad Organizacional. No obstante, dentro del liderazgo se agruparon también los ítems de supervisión. Lo cual refleja que la relación con sus superiores tiene un mismo significado para los empleados, $\mathrm{y}$ que la supervisión va acompañada de una buena relación con los superiores.

Tabla 2. Fiabilidad y Validez del Clima organizacional

\begin{tabular}{|c|c|c|c|c|c|c|}
\hline Ítems & $\begin{array}{l}\text { Liderazgo y } \\
\text { supervisión }\end{array}$ & Cohesión & $\begin{array}{c}\text { Factor } \\
\text { Ergonomía }\end{array}$ & $\begin{array}{l}\text { Presión } \\
\text { Laboral }\end{array}$ & $\begin{array}{c}\text { Claridad } \\
\text { Organizacional } \\
\end{array}$ & $\begin{array}{l}\text { Alpha } \\
\text { Cronbach }\end{array}$ \\
\hline $\begin{array}{l}\text { LI4 Facilidad para hablar con superior } \\
\text { sobre problemas de trabajo }\end{array}$ & 0.849 & & & & & 0.958 \\
\hline LI5 Apoyo en toma de decisiones & 0.839 & & & & & \\
\hline LI1 Respaldo total en acciones & 0.827 & & & & & \\
\hline LI2 Estímulo p/compartir ideas & 0.824 & & & & & \\
\hline $\begin{array}{l}\text { LI3 Captar sugerencias y hacer uso } \\
\text { constructivo de ellas }\end{array}$ & 0.816 & & & & & \\
\hline S4 Superior amable & 0.786 & & & & & \\
\hline $\begin{array}{l}\text { S2 Comprensión del jefe hacia sus } \\
\text { subordinados }\end{array}$ & 0.778 & & & & & \\
\hline $\begin{array}{l}\text { S3 Preocupación del jefe por la calidad } \\
\text { del trabajo realizado }\end{array}$ & 0.612 & & & & & \\
\hline $\begin{array}{l}\text { S5 Evaluación correcta sobre el } \\
\text { desempeño de funciones }\end{array}$ & 0.554 & & & & & \\
\hline $\begin{array}{l}\text { S1 Apoyo del superior para resolver } \\
\text { problemáticas }\end{array}$ & 0.511 & & & & & \\
\hline C1 Apoyo entre los trabajadores & & 0.745 & & & & 0.92 \\
\hline $\begin{array}{l}\text { C2 Buena aceptación de los compañeros } \\
\text { en el área }\end{array}$ & & 0.741 & & & & \\
\hline C3 Trabajo en equipo & & 0.697 & & & & \\
\hline C5 Colaboración eficaz & & 0.659 & & & & \\
\hline $\begin{array}{l}\text { C4 Disposición para compartir } \\
\text { información }\end{array}$ & & 0.593 & & & & \\
\hline ER5 Ambiente físico seguro & & & 0.737 & & & 0.815 \\
\hline ER4 Ventilación adecuada & & & 0.696 & & & \\
\hline ER3 Iluminación adecuada & & & 0.671 & & & \\
\hline ER1 Actividades de mantenimiento & & & 0.557 & & & \\
\hline ER2 Libre de contaminación auditiva & & & 0.453 & & & \\
\hline
\end{tabular}


PL4 Sentimiento de no descanso

0.874

0.823

PL3 Temor por llamadas

0.759

PL5 Estrés por exigencias

0.707

PL1 Excesiva carga de trabajo

0.572

CO2 Responsabilidad y esfuerzo esperado claramente definidos

0.840

0.840

CO1 Definición clara de cómo hacer el

trabajo

0.761

CO3 Normas de desempeño bien entendidas y comunicadas

0.506

Como se observa en la tabla anterior, la fiabilidad de la escala es adecuada al lograr un Alpha de Cronbach en las 5 dimensiones definidas superior al 0.700 que se considera como el umbral óptimo de la fiabilidad (Nunally, 1978).

A partir de los resultados anteriores, se acepta parcialmente la Hipótesis 1 que implica que las dimensiones que componen el clima organizaciones en una institución pública son: Liderazgo, Supervisión, Cohesión, Ergonomía, Presión Laboral y Claridad Organizacional. Lo anterior debido a que de acuerdo con el análisis factorial y las pruebas de fiabilidad las dimensiones que componen al clima organizacional son Liderazgo, Cohesión, Ergonomía, Presión Laboral y Claridad Organizacional, sin embargo, no se pudo demostrar que la Supervisión sea una dimensión valida del Clima Organizacional en una institución pública durante tiempos de pandemia del COVID-19.

Un análisis descriptivo de las dimensiones resultantes de la medición del clima organizacional puede observarse en el gráfico5.

Gráfica 5. Promedio de las puntuaciones de las dimensiones del clima organizacional

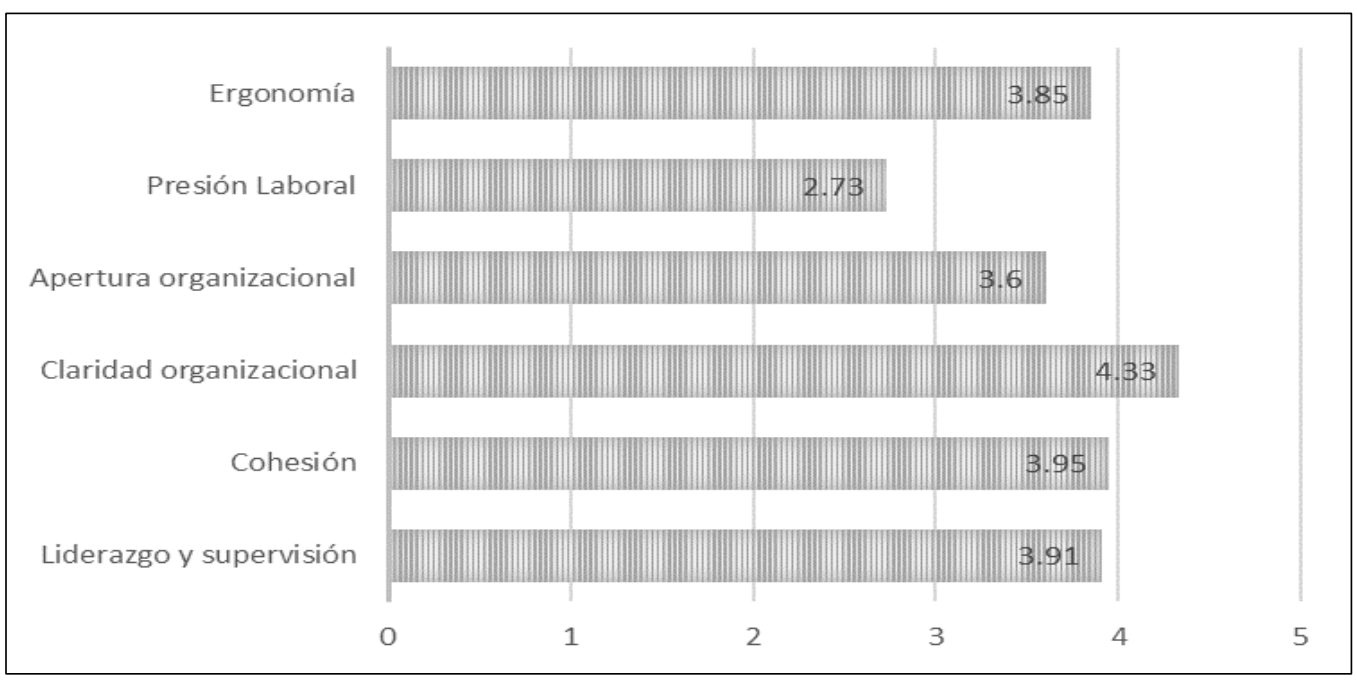

Fuente: elaboración propia a partir de datos recabados. 
Dicha gráfica permite analizar como la presión laboral es la dimensión del clima con una valoración más baja para los empleados, en este sentido los ítems que componen esta dimensión reflejan la situación de estrés y exigencias de trabajo altas percibidas por el empleado, consecuencia de las condiciones del entorno COVID, que llevan al trabajador a considerar que la carga laboral se ha visto ampliada y que no son capaces de cubrirlas en los tiempos marcados por la jornada laboral. Esto es compensado en cierta medida con la claridad organizacional percibida, puesto que es la dimensión más valorada dentro del clima organizacional, sin embargo, el hecho de que se tengan las responsabilidades y las normas de desempeño tan definidas puede llevar a los trabajadores a considerar una presión laboral superior, ya que los resultados esperados son altos.

\section{Conclusiones}

El clima organizacional se ha convertido en un elemento de la literatura de capital humano de mayor estudio en las últimas décadas, no obstante, no existe una definición clara de las dimensiones que componen este multiconstructo. Del mismo modo, y a raíz de los entornos turbulentos que enfrentamos actualmente el clima organizacional se vuelve determinante para comprender el desempeño de los trabajadores. La pandemia COVID-19 trajo consigo grandes retos para las organizaciones, tanto públicas como privadas. Por ello el objetivo de esta investigación era identificar las dimensiones del clima organizacional que se encuentran presentes ante esta situación de incertidumbre del entorno.

Los resultados obtenidos dejan entrever que el clima organizacional en tiempos de COVID-19 se identifica con 5 grandes dimensiones, Liderazgo y supervisión, cohesión, apertura organizacional, ergonomía, claridad organizacional y presión laboral.

En esta investigación se encontró que en tiempos de contingencial la presión laboral de los empleados es alta y que conlleva un mayor estrés que puede repercutir en su desempeño dentro de la organización. 


\section{Referencias}

Arellano Gault, D., \& Cabrero Mendoza, E. (2005). La Nueva Gestión Pública y su teoría de la organización: ¿son argumentos antiliberales? Justicia y equidad en el debate organizacional público. Gestión y Política Pública. XIV(3), 599-618. En http://hdl.handle.net/11651/1749

Argyris, C. (1958). Some Problems in Conceptualizing Organizational Climate: A Case Study of a Bank. Administrative Science Quarterly. 2(4), 501-520. En: https://www.jstor.org/stable/2390797?seq=1

Brown, S. P., \& Leigh, T. W. (1996). A new look at psychological climate and its relationship to job involvement, effort, and performance. Journal of applied psychology, 81(4), 358-368. En: https://psycnet.apa.org/doiLanding?doi=10.1037\%2F0021-9010.81.4.358

Brunet, L. (2005). El clima de trabajo en las organizaciones. Trillas.

Chaplin, J. P. \& Krawiec, T. S. (1968). Systems and theories of psychology. Holt, Rinehart \& Winston.

Chiang Vega, M. M., Salazar Botello, C. M., Huerta Rivera, P. C., \& Nuñez Partido, A. (2008). Clima organizacional y satisfacción laboral en organizaciones del sector estatal (Instituciones públicas) Desarrollo, adaptación y validación de instrumentos. Universum (Talca), 23(2), 66-85. En: http://dx.doi.org/10.4067/S0718-23762008000200004

Chiavenato, I. (2000). Administración de recursos humanos. Mc Graw Hill.

Christensen, T., Lægreid, P., \& Wise, L. R. (2002). Transforming administrative policy. Public Administration, 80(1), 153-178. En: https://doi.org/10.1111/1467-9299.00298

Dastmalchian, A., Adamson, R., \& Blyton, P. (1986). Developing a Measure of Industrial Relations Climate. Relations Industrielles / Industrial Relations, 41(4), 851-859. En: http://www.jstor.org/stable/23073121

Forehand, G. A., \& Von Haller, G. (1964). Environmental variation in studies of organizational behavior. Psychological Bulletin, 62(6), 361-382. En: https://doi.org/10.1037/h0045960

Freire, R. P. D. A. S., Martínez, J. M. D. L. T., Hernández, A. M. L., \& Paiva, S. B. (2011). El Clima Organizacional en el Diseño del Balanced Scorecard: Evaluación Psicométrica de un Instrumento de Medida. Contabilidad de Vista \& Revista, 22(1), 107-141. En: https://www.redalyc.org/pdf/1970/197017517005.pdf

García Solarte, M. (2009). Clima Organizacional y su Diagnóstico: Una aproximación Conceptual. Cuadernos de Administración, 25(42), 43-61. https://dialnet.unirioja.es/servlet/articulo?codigo $=5006497$ 
Velázquez, M. D. R. G., Gracia, T. J. H., Zorrilla, D. M. N., García, A. C., \& Monjaraz, G. S. (2014). Elementos de los modelos determinantes del clima organizacional. European Scientific Journal. 10(28), 107-122. En: https://core.ac.uk/download/pdf/236413706.pdf

Gan, F. (2007). El clima en las organizaciones. En Tejeda, J. \& Giménez, V. Formación de formadores (pp. 183-230). Thomson Editores.

Griffin, M. A., \& Curcuruto, M. (2016). Safety climate in organizations. Annual Review of Organizational Psychology and Organizational Behavior, 3(1), 191-212. En: https://doi.org/10.1146/annurevorgpsych-041015-062414

Hall, D. \& Schneider, B. (1972). Correlates of organizational identification as a function of career pattern and organizational type. In Academy of Management Proceedings (Vol. 1972, No. 1, pp. 159-161). Briarcliff Manor, NY 10510: Academy of Management. En: https://doi.org/10.5465/ambpp.1972.4981421

Hellriegel, D., \& Slocum Jr, J. W. (1974). Organizational climate: Measures, research and contingencies. Academy of management Journal, 17(2), 255-280. En: https://doi.org/10.5465/254979

Hernández, R; Fernández, C. \& Baptista, P. (2006). Metodología de la Investigación. Mc Graw Hill.

Hilton, T. (1966). Executive Leadership and Development. En Gilmer, B. y otros. Industrial psychology (pp. 228-253). McGraw-Hill.

Koys, D. J., \& DeCotiis, T. A. (1991). Inductive measures of psychological climate. Human relations, 44(3), 265-285. En: https://doi.org/10.1177/001872679104400304

Kuenzi, M., \& Schminke, M. (2009). Assembling fragments into a lens: A review, critique, and proposed research agenda for the organizational work climate literature. Journal of management, 35(3), 634717. En https://doi.org/10.1177/0149206308330559

Lewin, K., Lippitt, R., \& White, R. K. (1939). Patterns of aggressive behavior in experimentally created "social climates". The Journal of social psychology, 10(2), 269-299. En: https://doi.org/10.1080/00224545.1939.9713366

Likert, R., \& Likert, J. G. (1976). New ways of managing conflict. McGraw-Hill.

Litwin, G. \& Stringer, R. (1968), Motivation and Organizational Climate. Harvard University Press.

Martín Bris, M. (2000). Clima de trabajo y organizaciones que aprenden. Educar, (27), 103-117. En: https://www.raco.cat/index.php/Educar/article/view/20736

Moran, E. T., \& Volkwein, J. F. (1992). The cultural approach to the formation of organizational climate. Human relations, 45(1), 19-47. En: https://doi.org/10.1177/001872679204500102

Münch, L., \& Angeles, E. (2007). Métodos y técnicas de investigación. Trillas

Nicola, M., Alsafi, Z., Sohrabi, C., Kerwan, A., Al-Jabir, A., Iosifidis, C., Agha, M., \& Agha, R. (2020). The socio-economic implications of the coronavirus pandemic (COVID-19): A review. International Journal of Surgery, 78, 185-193. En: https://doi.org/10.1016/j.ijsu.2020.04.018

Nunnally, J. (1978). Psychometric theory. McGraw-Hill.

Patterson, M. G., West, M. A., Shackleton, V. J., Dawson, J. F., Lawthom, R., Maitlis, S., ... \& Wallace, A. M. (2005). Validating the organizational climate measure: links to managerial practices, productivity and innovation. Journal of organizational behavior, 26(4), 379-408. En: https://doi.org/10.1002/job.312

Payne, R. \& Pugh, D. (1976). Organizational structure and climate. En Dunnette, M. Handbook of industrial and organizational psychology (pp. 1125-1173). Rand McNally.

Pettigrew, A. M. (1979). On studying organizational cultures. Administrative science quarterly, 24(4), 570581. En: https://doi.org/10.2307/2392363

Pettigrew, A. M. (1990). Organizational climate and culture: two constructs in search of a role. En Schneider, B. Organizational climate and culture (pp. 413-434). Jossey-Bass Ltd.

Reichers, A. E. (1987). An interactionist perspective on newcomer socialization rates. Academy of management review, 12(2), 278-287. En https://doi.org/10.5465/amr.1987.4307838

Reichers, A. E. and Schneider, B. (1990). Climate and Culture: An Evolution of Constructs. En Schneider, B. Organizational climate and culture (pp. 5-39). Jossey-Bass Ltd. 
Segredo Pérez, A. M. (2013). Clima organizacional en la gestión del cambio para el desarrollo de la organización. Revista cubana de salud pública, 39(2), 385-393. En: http://scielo.sld.cu/scielo.php?script=sci_arttext\&pid=S086434662013000200017\&lng=es\&tlng=es.

Schneider, B. (1975). Organizational climates: An essay 1. Personnel psychology, 28(4), 447-479. En: https://doi.org/10.1111/j.1744-6570.1975.tb01386.x

Schneider, B., \& Reichers, A. E. (1983). On the etiology of climates. Personnel psychology, 36(1), 19-39. En: https://doi.org/10.1111/j.1744-6570.1983.tb00500.x

Schneider, B. (1987). The people make the place. Personnel psychology, 40(3), 437-453. En: https://doi.org/10.1111/j.1744-6570.1987.tb00609.x

Schneider, B., Ehrhart, M. G., \& Macey, W. H. (2013). Organizational climate and culture. Annual review of psychology, 64, 361-388. En: https://doi.org/10.1146/annurev-psych-113011-143809

Suprapti, S., Asbari, M., Cahyono, Y., \& Mufid, A. (2020). Leadership style, organizational culture and innovative behavior on public health center performance during pandemic covid-19. Journal of Industrial Engineering \& Management Research, 1(2), 76-88. En: https://doi.org/10.7777/jiemar.v1i2.42

Tagiuri, R., Litwin, G. H., \& Barnes, L. B. (1968). Organizational climate: Explorations of a concept. Division of Research, Graduate School of Business Administration, Harvard University.

Tiffin, J., \& McCormick, E. J. (1965). Industrial psychology. Prentice-Hall.

Toro Álvarez, F., \& Sanín Posada, A. (2013). Gestión del clima organizacional: intervención basada en evidencias. Centro de Investigación en Comportamiento Organizacional (CINCEL).

Viteles, M. S. (1953). Motivation and morale in industry. W. W. Norton. 